\title{
THE BIRDS NORTH OF LESSER SLAVE LAKE, ALBERTA
}

\author{
by HUGH C. SMITH*
}

The area north of Lesser Slave Lake, Alberta, has only recently been opened to vehicular travel and, as a result, ornithological investigations have been few. Erskine was the first to go into the area by car and report his findings. ${ }^{5}$ Early naturalists through the area, Preble, ${ }^{7}$ Williams ${ }^{19}$ and Eaton ${ }^{3}$, did not travel far from the two major rivers that border the area, the Peace on the west and north and the Athabasca on the east. Baker and Walkinshaw', Soper" and Godfrey" reported on their work in the area south of Lesser Slave Lake.

The Provincial Museum of Alberta spent 52 days during the last 4 years inventorying birds in the area. Camps were set up at Utikuma Lake (S4Tp80-R8W5) from June 1 to 6 and at a small lake, locally known as Bear Lake, (84-9W5) from June 14 to 20 in 1971; at Goosegrass Lake (91-3W5) from May 29 to June 10, 1972, from June 4 to 12 and June 21 to 30,1973 , and from May 28 to June 3, 1974. From here trips were made to God's Lake and Skunk Lake 5 and 2 miles ( 8 and $3 \mathrm{~km}$ ) away. The study area extended 75 miles $(120 \mathrm{~km})$ northeast of Utikuma Lake and was 2 to 3 miles (4 to $5 \mathrm{~km}$ ) wide. Erskine's study area was between Salt Prairie and Gift Lake. (Fig. 1).

\footnotetext{
*Provincial Museum of Alberta, Edmonton, Alta.

Provincial Museum of Alberta, Contribution No. 22
}

The study area is within the mixedwood section of the Boreal Forest Region. ${ }^{\text {"The }}$ major trees are Trembling Aspen (Populus tremuloides), Balsam Poplar (Populus balsamifera), White Spruce (Picea glauca), Black Spruce (Picea mariana), and Balsam Fir (Abies balsamea). Forest fires have occurred in most of the region at one time or another, so that in many places there is a large amount of deadfall which makes walking through the forest extremely difficult. Two oilfields, Nipisi and Red Earth, are located within the study area. As a result large sections of the forest are crossed by survey lines. Lakes of various sizes are found throughout the forest. The area is slightly rolling with no major topographic features. Trout Mountain, at an elevation of approximately 2,600 feet above sea level, is the highest point. The drainage is poor. Several small, slow moving streams connect many of the lakes and eventually drain into the Wabasca River which empties into the Peace River.

A number of people from the Provincial Museum were involved in field trips to the study area and their contribution to this paper is appreciated. Ludo Bogaert, Rod Burns, Michael Hampson, Jasper Keizer, Ron Solkoski, and David Spalding made their field notes available.

SPECIES LIST: The nomenclature used in this list (Table 1) is according to the 


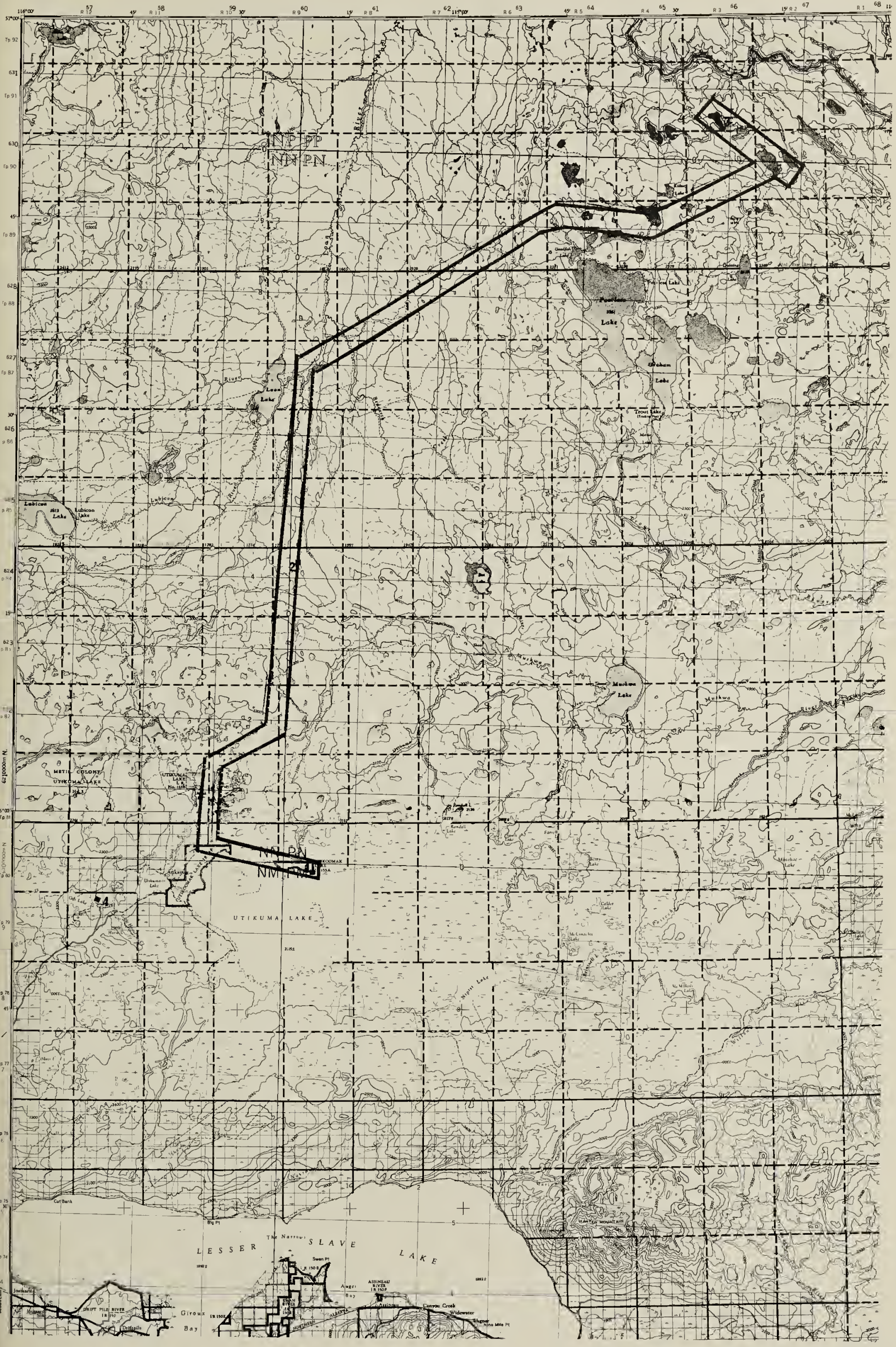

Fig. 1. Map of North Central Alberta, showing approximate route and the following camps: (1) Utikuma Lake Camp: (2) Bear Lake Camp: (3) Goosegrass Lake area; (4) Erskine’s Camp. 
Table 1

Species list for Uitkuma Lake - Goosegrass Lake area

\section{Species}

Common Loon*

Red-necked Grebe* $†$

Horned Grebe

Eared Grebe†

Western Grebet

Pied-billed Grebe†

White Pelican

Double-crested Cormorant

Great Blue Heron

American Bittern

Canada Goose

(Seen along Wabasca River)

Mallard* $\dagger$

Gadwall

Pintail

Green-winged Teal* $†$

Blue-winged Teal $\uparrow$

American Wigeon* $\dagger$

Northern Shovelert

Redhead $\dagger$

Ring-necked Duck†

Canvasback $\dagger$

Lesser Scaup $\dagger$

Common Goldeneye* $\dagger$

Barrow's Goldeneye

Bufflehead* $\dagger$

White-winged Scotert

Surf Scoter

Ruddy Duck $\dagger$

Common Merganser

(Seen along Wabasca River)

Goshawk

Cooper's Hawk

Sharp-shinned Hawk

Red-tailed Hawk $\dagger$

Bald Eagle*

Marsh Hawk

Osprey

American Kestrel $\dagger$

Spruce Grouse†

Ruffed Grouse $\dagger$

Sharp-tailed Grouse $†$

Sora

American $\mathrm{Coot}^{*} \dagger$

Killdeer*

Common Snipe

\begin{tabular}{|c|c|c|c|c|c|c|c|}
\hline \multirow[b]{2}{*}{ 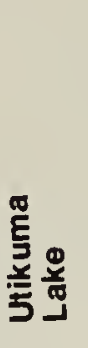 } & \multirow[b]{2}{*}{ 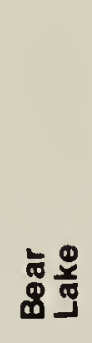 } & \multirow{2}{*}{ 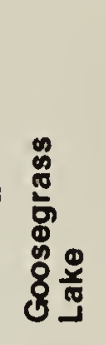 } & \multirow[b]{2}{*}{ 离 } & \multirow[b]{2}{*}{ 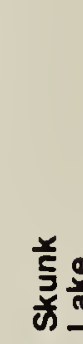 } & \multirow{2}{*}{ 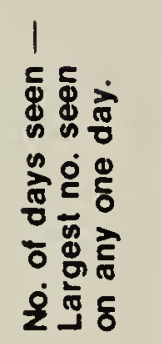 } & \multicolumn{2}{|c|}{$\begin{array}{l}\% \text { of days } \\
\text { seen }\end{array}$} \\
\hline & & & & & & $\begin{array}{l}\stackrel{g}{\frac{5}{2}} \\
\frac{\omega}{2}\end{array}$ & 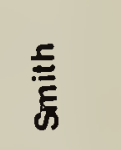 \\
\hline $\mathrm{X}$ & $\mathrm{X}$ & $\mathrm{x}$ & & $\mathrm{x}$ & $14-3$ & 40.9 & 26.9 \\
\hline & $\mathrm{x}$ & $\mathrm{x}$ & & $\mathrm{x}$ & $22-6$ & 56.8 & 42.3 \\
\hline $\mathrm{X}$ & & $\mathrm{X}$ & & $\mathrm{x}$ & $5-4$ & 50.0 & 9.6 \\
\hline $\mathrm{x}$ & $\mathrm{X}$ & & & & $2-2$ & 11.4 & 3.8 \\
\hline $\mathrm{x}$ & & & $\mathrm{X}$ & & $4-25$ & 6.8 & 7.7 \\
\hline & $\mathrm{x}$ & & & & $3-2$ & 11.4 & 5.8 \\
\hline $\mathrm{X}$ & & & & & $1-1$ & 2.3 & 1.9 \\
\hline & & $\mathrm{X}$ & & & $1-1$ & & 1.9 \\
\hline & & $\mathrm{x}$ & & & $4-2$ & $\begin{array}{l}2.3 \\
2.3\end{array}$ & 7.7 \\
\hline & & & & & $3-4$ & & 5.8 \\
\hline $\mathrm{x}$ & $\mathrm{x}$ & $\mathrm{x}$ & $\mathrm{x}$ & $\mathrm{x}$ & $22-17$ & 61.4 & 42.3 \\
\hline & $\mathrm{x}$ & $\mathrm{x}$ & $\mathrm{x}$ & & $6-2$ & 6.8 & 11.5 \\
\hline $\mathrm{X}$ & & & $\mathrm{x}$ & & $2-7$ & 9.1 & 3.8 \\
\hline $\mathrm{x}$ & & $\mathrm{x}$ & & $\mathrm{x}$ & $9-5$ & 47.7 & 17.3 \\
\hline $\mathrm{x}$ & & $\mathrm{x}$ & & $\mathrm{x}$ & $13-5$ & 50.0 & 25.0 \\
\hline $\mathrm{x}$ & $\mathrm{x}$ & $\mathrm{x}$ & $\mathrm{X}$ & $\mathrm{x}$ & $17-21$ & 61.4 & 32.7 \\
\hline $\mathrm{x}$ & $\mathrm{x}$ & $\mathrm{x}$ & & $\mathrm{x}$ & $5-6$ & 18.2 & 9.6 \\
\hline $\mathrm{x}$ & & & & & $1-1$ & 20.5 & 1.9 \\
\hline & $\mathrm{x}$ & $\mathrm{x}$ & & $\mathrm{x}$ & $3-5$ & 18.2 & 5.8 \\
\hline $\mathrm{x}$ & & & & & $1-1$ & 9.1 & 1.9 \\
\hline $\mathrm{x}$ & $\mathrm{x}$ & $\mathrm{x}$ & $\mathrm{X}$ & $\mathrm{x}$ & $15-32$ & 75.0 & 28.8 \\
\hline $\mathrm{X}$ & $\mathrm{x}$ & $\mathrm{x}$ & $\mathrm{X}$ & $\mathrm{x}$ & $16-30$ & $\begin{array}{l}52.3 \\
182\end{array}$ & 30.8 \\
\hline $\mathrm{x}$ & $\mathrm{x}$ & $\mathrm{x}$ & $\mathrm{X}$ & $\mathrm{x}$ & $18-100$ & 72.7 & 34.6 \\
\hline $\mathrm{x}$ & $\mathrm{x}$ & $\mathrm{x}$ & $\mathrm{x}$ & $\mathrm{x}$ & $22-24$ & 59.1 & 42.3 \\
\hline & $\mathrm{x}$ & $\mathrm{X}$ & & $\mathrm{x}$ & & $\begin{array}{r}2.3 \\
47.7\end{array}$ & $\begin{array}{l}7.7 \\
5.8\end{array}$ \\
\hline & & & & & $1-2$ & & 1.9 \\
\hline $\mathrm{x}$ & & $\mathrm{x}$ & $\mathrm{x}$ & & $4-1$ & $\begin{array}{l}2.3 \\
2.3\end{array}$ & 7.7 \\
\hline $\mathrm{x}$ & & & & & $3-2$ & & 5.8 \\
\hline $\mathrm{x}$ & $\mathrm{x}$ & $\mathrm{x}$ & & & $12-1$ & 38.6 & 23.1 \\
\hline & $\mathrm{x}$ & $\mathrm{x}$ & & & $14-4$ & 2.3 & 26.9 \\
\hline $\mathrm{X}$ & $\mathrm{x}$ & & $\mathrm{x}$ & & $7-4$ & 15.9 & 13.4 \\
\hline $\begin{array}{l}\mathrm{X} \\
\mathrm{x}\end{array}$ & $\mathrm{x}$ & $\mathrm{x}$ & & $x$ & $\begin{array}{r}3-2 \\
11-4\end{array}$ & 568 & 21.8 \\
\hline & & & & & & 4.5 & \\
\hline $\mathrm{x}$ & $\mathrm{x}$ & $\mathrm{x}$ & $\mathrm{x}$ & $\mathrm{x}$ & $8-1$ & $\begin{array}{r}13.6 \\
2.3\end{array}$ & 15.4 \\
\hline $\mathrm{x}$ & $\mathrm{x}$ & $\mathrm{x}$ & & $\mathrm{X}$ & $9-1$ & 31.8 & 17.3 \\
\hline $\mathrm{x}$ & $\mathrm{x}$ & $\mathrm{x}$ & & $\mathrm{x}$ & $12-3$ & 65.9 & 23.1 \\
\hline $\mathrm{X}$ & $\mathrm{x}$ & $\mathrm{x}$ & & & 8-2 & 56.8 & 15.4 \\
\hline $\mathrm{x}$ & & $\mathrm{x}$ & $x$ & $\mathrm{x}$ & $22-4$ & 47.7 & 42.3 \\
\hline
\end{tabular}

${ }^{*}$ Evidence of nesting in this study.

tEvidence of nesting in Erskine's study. 
Upland Plover

Spotted Sandpiper*

Solitary Sandpiper†

Greater Yellowlegs

Lesser Yellowlegs

Baird's Sandpiper

Pectoral Sandpiper

Least Sandpiper

Dowitcher

Semipalmated Sandpiper

Northern Phalarope

Wilson's Phalarope

Herring Gull

California Gull

Ring-billed Gull

Franklin's Gull $\dagger$

Bonaparte's Gull $\dagger$

Common Tern $\dagger$

Black Tern†

Rock Dovet

Mourning Dove

Great Horned Owl

Hawk Owl

Long-eared Owl

Short-eared Owl

Common Nighthawk

Belted Kingfisher

Common Flicker* $\dagger$

Pileated Woodpecker

Yellow-bellied Sapsucker* $†$

Hairy Woodpecker

Downy Woodpecker $\dagger$

Northern Three-toed

Woodpecker

Eastern Kingbird* $†$

Eastern Phoebe

Say's Phoebe

Alder Flycatcher

Least Flycatcher*

Western Wood Pewee*

Olive-sided Flycatcher

Horned Lark

Tree Swallow

Bank Swallow

Barn Swallow $\dagger$

Cliff Swallow

(Colony seen at

Red Earth)

Purple Martin

Gray Jay

Blue Jay

Black-billed Magpie

Common Raven

Common Crow

Black-capped Chickadee $\dagger$

Boreal Chickadee*

White-breasted Nuthatch

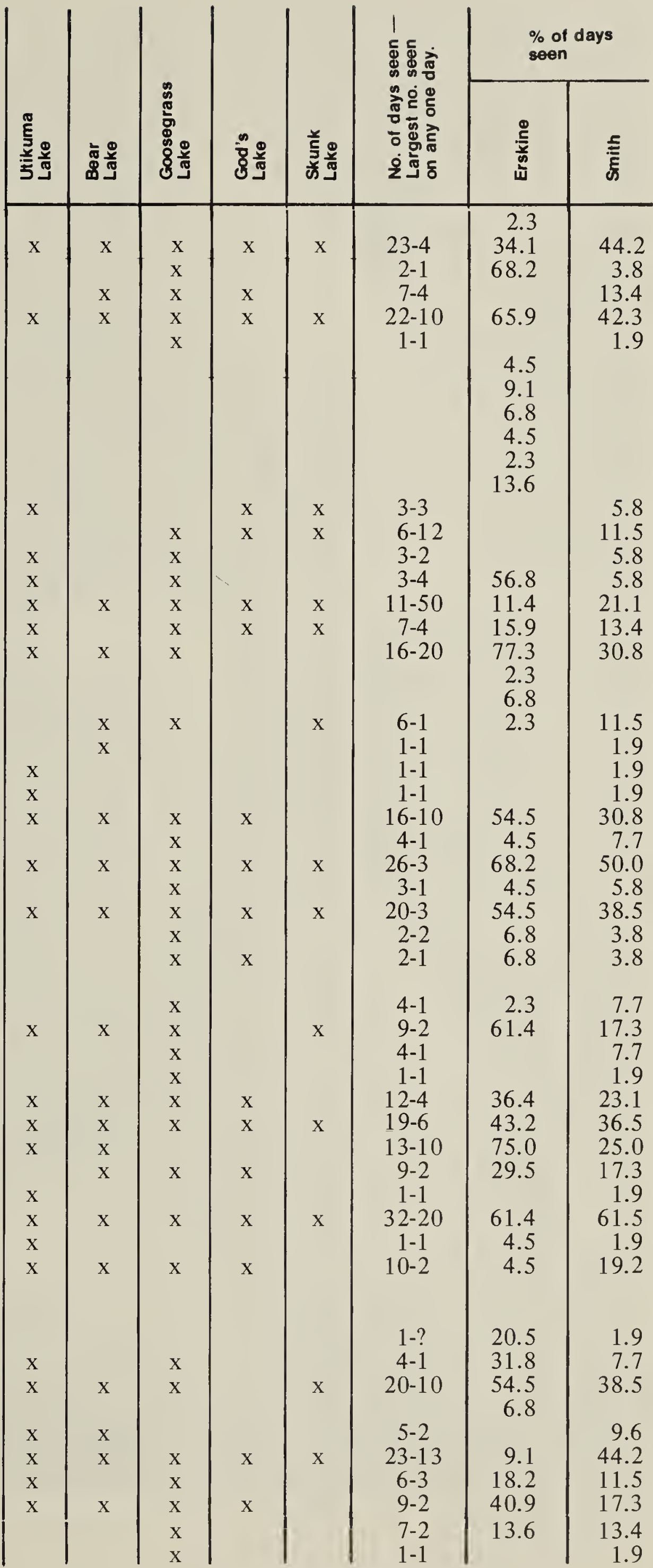




\section{Species}

Red-breasted Nuthatch* Brown Creeper

House Wren

Winter Wren

Long-billed Marsh Wren (Seen at Loon Lake) Gray Catbird American Robin* $†$ Hermit Thrush* Swainson's Thrush* $\dagger$ Mountain Bluebird Golden-crowned Kinglet Ruby-crowned Kinglet Bohemian Waxwing Cedar Waxwing Common Starling* $†$ Solitary Vireo Red-eyed Vireo* Philadelphia Vireo Warbling Vireo

Black-and-White Warbler Tennessee Warbler* Yellow Warbler Magnolia Warbler Cape May Warbler Yellow-rumped Warbler Black-throated Green Warbler Bay-breasted Warbler Blackpoll Warbler Palm Warbler

Ovenbird

Northern Waterthrush Connecticut Warbler Mourning Warbler Common Yellowthroat Wilson's Warbler Canada Warbler American Redstart House Sparrow Yellow-headed Blackbird Red-winged Blackbird* + Northern Oriole Rusty Blackbird* Brewer's Blackbird Common Grackle† Brown-headed Cowbird Western Tanager Rose-breasted Grosbeak Evening Grosbeak Purple Finch

Pine Grosbeak

Pine Siskin

American Goldfinch White-winged Crossbill Savannah Sparrow LeConte's Sparrow Sharp-tailed Sparrow

\begin{tabular}{|c|c|c|c|c|c|c|c|}
\hline \multirow[b]{2}{*}{ 造 } & \multirow[b]{2}{*}{ 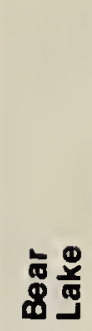 } & \multirow{2}{*}{ 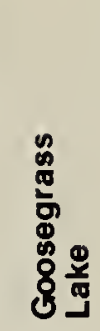 } & \multirow[b]{2}{*}{ 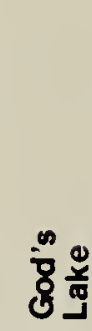 } & \multirow[b]{2}{*}{ 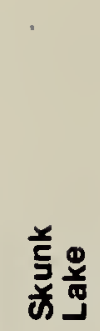 } & \multirow{2}{*}{ 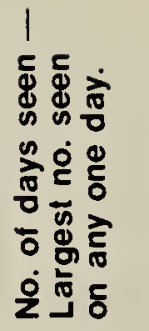 } & \multicolumn{2}{|c|}{$\begin{array}{l}\% \text { of days } \\
\text { soen }\end{array}$} \\
\hline & & & & & & 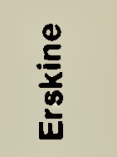 & 站 \\
\hline & & $\begin{array}{l}\mathrm{x} \\
\mathrm{x}\end{array}$ & & & $\begin{array}{r}10-3 \\
4-3\end{array}$ & $\begin{array}{r}2.3 \\
18.2 \\
9.1\end{array}$ & $\begin{array}{r}19.2 \\
7.7\end{array}$ \\
\hline & & $\mathrm{x}$ & & & $\begin{array}{l}1-1 \\
1-1\end{array}$ & 9.1 & 1.9 \\
\hline $\mathrm{x}$ & $\mathrm{x}$ & $\mathrm{x}$ & $\mathrm{x}$ & $\mathrm{x}$ & $23-4$ & 90.1 & 44.2 \\
\hline $\mathrm{x}$ & & $\mathrm{x}$ & $\mathrm{x}$ & $\mathrm{x}$ & $10-2$ & 22.7 & 19.2 \\
\hline $\mathrm{x}$ & $\mathrm{x}$ & $\mathrm{x}$ & $\mathrm{x}$ & $\mathrm{x}$ & $30-6$ & $\begin{array}{r}43.2 \\
2.3 \\
2.3\end{array}$ & 57.7 \\
\hline & $\mathrm{x}$ & $\mathrm{x}$ & $\mathrm{x}$ & & $12-9$ & 15.9 & 23.1 \\
\hline & $\mathrm{x}$ & $\mathrm{x}$ & & & $5-5$ & & 9.6 \\
\hline $\begin{array}{l}\mathrm{x} \\
\mathrm{x}\end{array}$ & $\mathrm{x}$ & $\mathrm{x}$ & & & $\begin{array}{l}3-6 \\
3-2\end{array}$ & $\begin{array}{l}47.7 \\
34.1\end{array}$ & $\begin{array}{l}5.8 \\
5.8\end{array}$ \\
\hline & & $\mathrm{x}$ & & & $5-\overline{5}$ & & 9.6 \\
\hline $\mathrm{x}$ & $\mathrm{x}$ & $\begin{array}{l}\mathrm{x} \\
\mathrm{x}\end{array}$ & $\mathrm{x}$ & $\mathrm{x}$ & $\begin{array}{r}20-6 \\
3-2\end{array}$ & 34.1 & $\begin{array}{r}38.5 \\
5.8\end{array}$ \\
\hline $\mathrm{x}$ & $\mathrm{x}$ & & $\mathrm{x}$ & & 4-2 & 28.6 & 7.7 \\
\hline $\mathrm{x}$ & & $\mathrm{x}$ & $\mathrm{x}$ & & $11-3$ & 22.7 & 21.1 \\
\hline $\mathrm{x}$ & $\mathrm{x}$ & $\mathrm{x}$ & $\mathrm{x}$ & $\mathrm{x}$ & $45-17$ & 28.6 & 86.5 \\
\hline $\mathrm{x}$ & & $\mathrm{x}$ & & $\mathrm{x}$ & $14-9$ & 38.6 & 26.9 \\
\hline $\mathrm{x}$ & & $\begin{array}{l}x \\
x\end{array}$ & $\mathrm{x}$ & & $\begin{array}{l}4-4 \\
3-6\end{array}$ & $\begin{array}{l}2.3 \\
2.3\end{array}$ & $\begin{array}{l}7.7 \\
5.8\end{array}$ \\
\hline $\mathrm{x}$ & & $\mathrm{x}$ & $\mathrm{x}$ & $\mathrm{x}$ & $21-13$ & $\begin{array}{r}18.2 \\
45\end{array}$ & 40.4 \\
\hline & & $\mathrm{x}$ & & & $8-25$ & 4.5 & 15.4 \\
\hline $\mathrm{x}$ & $\mathbf{y}$ & & $\mathrm{x}$ & $\mathrm{Y}$ & $2-1$ & 4.5 & $\begin{array}{l}3.8 \\
5.8\end{array}$ \\
\hline & $\begin{array}{l}\mathrm{A} \\
\mathrm{X}\end{array}$ & $\begin{array}{l}x \\
x\end{array}$ & $\mathrm{x}$ & $x$ & $\begin{array}{r}5-2 \\
12-8\end{array}$ & $\begin{array}{r}0.8 \\
38.6\end{array}$ & $\begin{array}{r}5.8 \\
23.1\end{array}$ \\
\hline $\mathrm{x}$ & & $\mathrm{x}$ & $\mathrm{x}$ & $\mathrm{x}$ & $16-3$ & 25.0 & 30.8 \\
\hline $\mathrm{x}$ & & & & & $1-1$ & 2.3 & 1.9 \\
\hline $\mathrm{x}$ & $\mathrm{x}$ & $\mathrm{x}$ & & & $9-5$ & 18.2 & 17.3 \\
\hline $\mathrm{x}$ & $\mathrm{x}$ & $\mathrm{x}$ & & & $9-2$ & 18.2 & 17.3 \\
\hline $\mathrm{x}$ & & $\mathrm{x}$ & & & $\begin{array}{l}1-1 \\
1-2\end{array}$ & & $\begin{array}{l}1.9 \\
1.9\end{array}$ \\
\hline $\mathrm{x}$ & & $\mathrm{x}$ & & & $6-5$ & 15.9 & 11.5 \\
\hline $\mathrm{x}$ & $\mathrm{x}$ & $\mathrm{x}$ & & & $5-1$ & $\begin{array}{r}28.6 \\
4.5\end{array}$ & 9.6 \\
\hline $\mathrm{x}$ & $\mathrm{x}$ & $\mathrm{x}$ & & $\mathrm{x}$ & $25-25$ & $\begin{array}{r}90.1 \\
2.3\end{array}$ & 48.1 \\
\hline & & $\mathrm{x}$ & $\mathrm{x}$ & $\mathrm{x}$ & $7-10$ & 15.9 & 13.4 \\
\hline $\mathrm{x}$ & $\mathrm{x}$ & & & $\mathrm{x}$ & $5-5$ & 11.4 & 9.6 \\
\hline & & $\mathrm{x}$ & & $\mathrm{x}$ & $5-4$ & 31.8 & 9.6 \\
\hline $\mathrm{X}$ & $\mathrm{x}$ & $\mathrm{x}$ & $\mathrm{x}$ & $\mathrm{x}$ & $10-25$ & 28.6 & 19.2 \\
\hline $\mathrm{x}$ & & $\mathrm{x}$ & & & $17-5$ & 2.3 & 32.7 \\
\hline $\mathrm{x}$ & $\mathrm{x}$ & $\mathrm{x}$ & $\mathrm{x}$ & & $16-3$ & 9.1 & 30.8 \\
\hline & & $\mathrm{x}$ & & & $2-4$ & 2.3 & 3.8 \\
\hline & $\mathrm{X}$ & $\begin{array}{l}x \\
x\end{array}$ & & & $\begin{array}{l}7-3 \\
2-1\end{array}$ & $\begin{array}{l}6.8 \\
2.3\end{array}$ & $\begin{array}{r}13.4 \\
3.8\end{array}$ \\
\hline & $\mathrm{x}$ & $\mathrm{x}$ & $\mathrm{x}$ & & $8-10$ & 22.7 & 15.4 \\
\hline $\mathrm{X}$ & & $\mathrm{x}$ & & & $2-1$ & 2.3 & 3.8 \\
\hline & $\mathrm{X}$ & & & & $2-2$ & 13.6 & 3.8 \\
\hline $\mathrm{X}$ & & & & & $5-1$ & 11.4 & 9.6 \\
\hline $\mathrm{x}$ & $\mathrm{X}$ & & $\mathrm{x}$ & $\mathrm{x}$ & $4-2$ & $\begin{array}{l}22.7 \\
11.4\end{array}$ & 7.7 \\
\hline
\end{tabular}




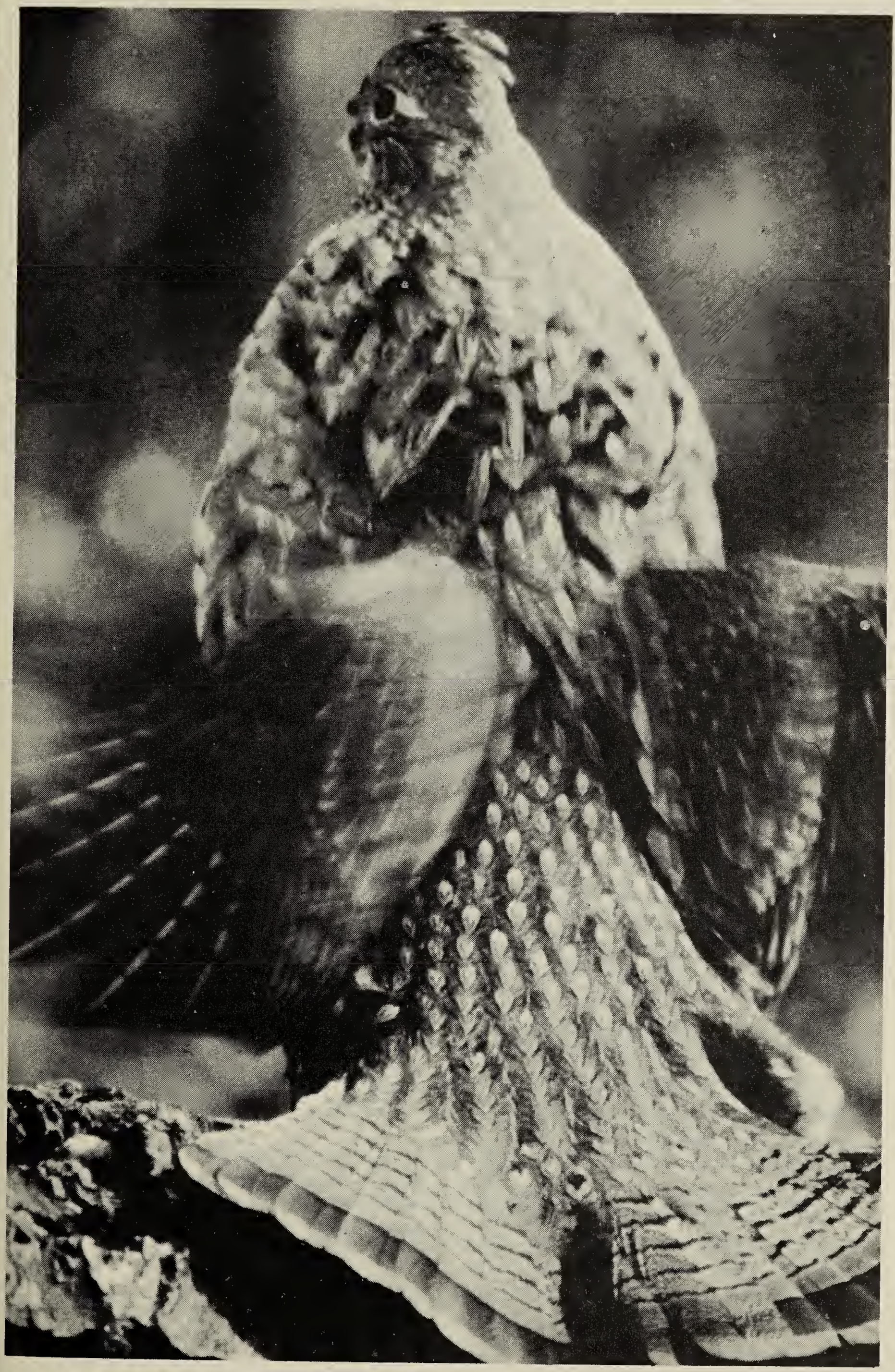

Ruffed Grouse drumming.

L. A. Morgotch 


\begin{tabular}{|c|c|c|c|c|c|c|c|c|}
\hline Vesper Sparrow & & & & & & & 6.8 & \\
\hline $\begin{array}{l}\text { Lark Sparrow (A flock seen } \\
\text { vicinity Trout Mtn.) }\end{array}$ & & & & & & $1-4$ & & 1.9 \\
\hline Dark-eyed Junco* $\dagger$ & & $\mathrm{x}$ & $\mathrm{x}$ & $\mathrm{x}$ & $\mathrm{x}$ & $21-8$ & 40.9 & 40.4 \\
\hline Chipping Sparrow* & $\mathrm{x}$ & $\mathrm{x}$ & $\mathrm{x}$ & $\mathrm{x}$ & $\mathrm{x}$ & $24-12$ & 34.1 & 46.2 \\
\hline Clay-colored Sparrow & $\mathrm{x}$ & & & & & & 15 & 11.5 \\
\hline White-throated Sparrow* & $\mathrm{x}$ & $\mathrm{x}$ & $\mathrm{x}$ & $\mathrm{X}$ & $\mathrm{x}$ & $44-7$ & 59.1 & 84.6 \\
\hline Fox Sparrow & & & $\mathrm{x}$ & & $\mathrm{x}$ & $4-1$ & 25.0 & 7.7 \\
\hline Lincoln' Sparrow & $\mathrm{x}$ & $\mathrm{x}$ & $\mathrm{x}$ & & $\mathrm{x}$ & $6-4$ & 47.7 & 11.5 \\
\hline Swamp Sparrow & $\mathrm{x}$ & & & $\mathrm{x}$ & $\mathrm{x}$ & $3-1$ & 28.6 & 5.8 \\
\hline Song Sparrow & $\mathrm{x}$ & $\mathrm{x}$ & $\mathrm{x}$ & & & $12-3$ & 40.9 & 23.1 \\
\hline
\end{tabular}

A.O.U. Checklist, as amended by Eisenmann. ${ }^{4}$ To provide a comprehensive list of the birds of the area I have included species recorded by Erskine.

DISCUSSION: This study recorded 140 species with evidence of nesting for 29. Erskine reported 138 species of which 44 were nesting. The two studies combined recorded 164 species with 58 nesting.

To indicate abundance Erskine reported the number of days a species was seen as well as the greatest number of individuals seen on a given day. For example, his notation "18-3" indicated that a species was seen on 18 days and that 3 individuals was the greatest number seen on one day. In order to compare Erskine's data with this study I used the same index and then converted the days seen to a percentage of possible days seen for both studies. I used 45 days for Erskine's study as determined from the dates listed in his paper.
The two studies disagree on the most frequently recorded species (Table 2). These differences may not be solely a factor of abundance but may be, and probably are, artifacts of the studies. For example, the way data was recorded, the skill of the observers in identifying birds, the diligence with which areas were searched for birds, and the amount of time spent in different habitats all tend to distort the number and frequency of birds recorded. As an illustration of this, it is tempting to say

\section{Table 2}

Five most frequently recorded species in decreasing order of frequency

Tennessee Warbler

$$
\text { SMITH }
$$

White-throated Sparrow

Tree Swallow

Swainson's Thrush

Common Flicker

American Robin and

\section{ERSKINE}

Red-winged Blackbird

Black Tern

Lesser Scaup

Western Wood Pewee 
that Robins and Red-winged Blackbirds were the most common birds of the area according to Erskine. This may be so but, if Erskine's camp was so situated that it was adjacent to a marsh or was within the nesting area of a Robin, these birds would have a tendency to be recorded more frequently than others. If, however, the recording of species was always done away from the camp site these data would have a different meaning.

\section{Table 3}

Six most frequently recorded ducks

Mallard

Wh.-wgd. Scoter

Bufflehead

Am. Wigeon

Com. Goldeneye

Lesser Scaup

$\begin{array}{cc}\text { Smith } & \text { Erskine } \\ 1 & 3 \\ 1 & 5 \\ 3 & 2 \\ 4 & 3 \\ 4 & 6 \\ 6 & 1\end{array}$

In spite of these inherent problems, it is interesting to note the similarity in the two studies if the birds are broken down into groups (Tables $3,4,5$ ). It

\section{Table 4}

Five most frequently recorded warblers

Tenn. Warbler

Yel.-rmpd. Warbler

N. Waterthrush

$\begin{array}{cc}\text { Smith } & \text { Erskine } \\ 1 & 3 \\ 2 & - \\ 3 & 4 \\ 4 & 1 \\ 5 & 2 \\ - & 5\end{array}$

Table 5

Five most freqently recorded fringillids
Wh.-thr. Sparrow
Chipping Sparrow
Dark-eyed Junco
Rose-br. Grosbeak
Song Sparrow
Lincoln's Sparrow

$\begin{array}{cc}\text { Smith } & \text { Erskine } \\ 1 & 1 \\ 2 & 5 \\ 3 & 3 \\ 4 & - \\ 5 & 4 \\ - & 2\end{array}$

will be interesting to see if changes occur in these groups as more studies are done in the area.

'AMERICAN ORNITHOLOGISTS' UNION. 1957. Check-list of North American Birds. Fifth Edition. $691 \mathrm{p}$.

${ }^{2}$ BAKER, B. W., and L. H. WALKINSHAW. 1946 Birds Notes from Fawcett, Alberta. Can. Field-Nat. 60:5-10.

${ }^{3}$ EATON, S. W. 1948. Bird distribution along the Peace, Slave and Little Buffalo Rivers of Canada. Auk. 65:345-352.

'EISENMANN, E. 1973. Thirty-second Supplement to the American Ornithologists' Union Check-list of North American Birds. Auk. 90:411-419.

"ERSKINE, A. J. 1968. Birds observed in north-central Alberta, summer 1964, Blue Jay 26;24-31.

"GODFREY, W. E. 1952. Birds of the Lesser Slave Lake-Peace River areas Alberta. Nat. Mus. Canada Bull. 126:142-175.

'PREBLE, E. A. 1908. A biological investigation of the Athabasca-Mackenzie region. U.S. Biol. Surv., N.A. Fauna, No. 27:574 p.

${ }^{\times}$ROWE, J. S. 1972. Forest regions of Canada. Dept. of the Environment, Can. Forestry Service Publ. No. $1300,172 \mathrm{p}$.

${ }^{9}$ SOPER, J. D. 1949. Birds observed in the Grande Prairie-Peace River region of northwestern Alberta, Canada. Auk 66:233-257.

"WILLIAMS, M. Y. 1922. Biological notes covering parts of the Peace, Liard, Mackenzie and Great Bear River basins. Can. Field-Nat. 47:23-31. 